\title{
Author Correction: Non-Abelian generalizations of the Hofstadter model: spin-orbit-coupled butterfly pairs
}

Yi Yang (D, Bo Zhen (D, John D. Joannopoulos and Marin Soljačić (D)

Correction to: Light: Science \& Applications https://doi.org/10.1038/s41377-020-00384-7

published online 19 October 2020

Eq. (3) of the originally published paper:

" $\boldsymbol{A}=\left(0, m \theta \sigma_{y}+m \phi \sigma_{z}, 0\right)$ " should be corrected to

" $\boldsymbol{A}=\left(0, \log \left[\exp \left(\mathrm{i} m \theta \sigma_{y}\right) \exp \left(\mathrm{i} m \phi \sigma_{z}\right)\right], 0\right) "$
This has been corrected in both the PDF and the HTML versions of the paper.

Published online: 07 April 2021 